\title{
Experiencia inicial con cirugía revisional bariátrica asistida por robot
}

\author{
James Hamilton S. ${ }^{1}$, Camila Onetto C. ${ }^{1}$,
} Omar Orellana E. ${ }^{1}$ y Pablo Marín P. ${ }^{1}$

\section{Initial experience with robotic assisted revisional bariatric surgery}

Background: Robotics in bariatric surgery is a novel development in medical institutions in Chile. Revisional bariatric procedures have increased during the last years. There are no publications in our country related to the initial experience and results in robotic revisional bariatric surgery. The aim of this study is to describe the initial experience and short term outcomes with robotic revisional bariatric surgery. Study Design: We conducted a retrospective study of all consecutive robotic revisional bariatric surgeries performed by a single surgeon in Clínica Santa María in Santiago, Chile. We analyzed demographic characteristics and surgical outcomes, morbidity, mortality and length of hospital stay. Results: 59 revisional bariatric surgeries were performed, 11 reshaping of sleeve gastrectomy, 34 Roux in Y gastric bypass, 1 pouch reshaping of Roux in Y gastric bypass, 3 extractions of laparoscopic adjustable gastric banding, 1 of them with simultaneous sleeve gastrectomy, 5 laparoscopic adjustable gastric banding to patients with previous Roux in Y gastric bypass, 3 total gastrectomy, 1 resection of gastro-gastric fistula and 1 gastro enteral anastomosis. Mean age was 47.8 years. Average preoperative body mass index was $33.86 \mathrm{~kg} / \mathrm{m}^{2}$. Mean surgical time was 101.63 minutes. Postoperative complications were observed in 3 patients $(5.08 \%)$. In $2(3.39 \%)$ of this patient, reoperation was required. There were no deaths in this group. Average length of hospital stay was 3.6 days. Conclusion: Robotic revisional bariatric surgery is a novel technique that appears to be safe and effective.

Key words: robotic assisted surgery; revisional bariatric surgery; bariatric surgery.

\section{Resumen}

Introducción: La cirugía bariátrica robótica es una técnica novedosa y en desarrollo en Chile. Los procedimientos bariátricos revisionales han aumentado durante los últimos años. No existen publicaciones en nuestro país relacionadas con experiencias en cirugía bariátrica revisional robótica. El objetivo de este estudio es describir la experiencia inicial y los resultados a corto plazo con el uso de la cirugía robótica en estos procedimientos. Material y Métodos: Realizamos una revisión retrospectiva de todos los pacientes sometidos a cirugía bariátrica revisional asistida por robot, en Clínica Santa María, de Santiago de Chile. Analizamos las características demográficas, resultados quirúrgicos, morbilidad, mortalidad y tiempo de estadía hospitalaria. Resultados: Se realizaron un total de 59 cirugías bariátricas revisionales asistidas por robot, 11 remodelamientos de gastrectomía en manga, 34 bypass gástrico en Y-Roux, 1 remodelamiento del pouch gástrico en un bypass gástrico en Y-Roux, 3 extracciones de banda gástrica ajustable, 1 de ellas con gastrectomía en manga simultánea, 5 instalaciones de banda gástrica ajustable en pacientes con bypass gástrico en Y-Roux, 3 gastrectomías totales, 1 resección de fístula gastro-gástrica y una anastomosis gastroyeyunal. La edad promedio fue de 47,8 años. El Índice de Masa Corporal promedio preoperatorio fue de $33,86 \mathrm{~kg} / \mathrm{m}^{2}$. El tiempo quirúrgico promedio fue de 101,63 min. Se observaron complicaciones postoperatorias en 3 pacientes $(5,08 \%)$. En $2(3,39 \%)$ de ellos, se requirió una reoperación. No hubo fallecidos. El tiempo promedio de estadía hospitalaria fue de 3,6 días. Conclusión: La cirugía bariátrica revisional asistida por robot es una técnica novedosa, que parece ser segura y efectiva.

Palabras clave: cirugía asistida por robot, cirugía bariátrica revisional, cirugía bariátrica. 


\section{Introducción}

La cirugía bariátrica sigue siendo el único tratamiento efectivo, comprobado, a largo plazo, para la baja de peso. El procedimiento realizado con mayor frecuencia a nivel mundial es el bypass gástrico en Y-Roux (BGYR) ${ }^{1}$.

En EE. UU. la gastrectomía en manga es el procedimiento bariátrico realizado con mayor frecuencia.

A pesar de lo descrito, teniendo buenos resultados a corto y mediano plazo con respecto a la baja de peso, el seguimiento a largo plazo ha demostrado una importante incidencia de reganancia de peso. La enfermedad por reflujo gastroesofágico intratable, es otro diagnóstico cuya incidencia ha aumentado, principalmente en pacientes con gastrectomía en manga ${ }^{2}$.

Para el manejo de la obesidad mórbida, el BGYR ha sido considerado el tratamiento de elección o estándar de oro por años. La banda gástrica ajustable (BGA) y la gastrectomía en manga, han sido consideradas alternativas al BGYR.

La BGA ha demostrado un bajo porcentaje de complicaciones postoperatorias, y porcentaje de baja de peso aceptable, sin embargo, se han reportado complicaciones a largo plazo, asociado a una importante reganancia de peso. El porcentaje de cirugía revisional asociado a procedimientos como la BGA y la gastrectomía en manga se encuentra entre 8 y $58 \%{ }^{3}$.

Se han descrito resultados de la gastrectomía en manga, a corto y mediano plazo, similares a los observados en pacientes con BGYR, sin embargo, los resultados a largo plazo demuestran una importante reganancia del peso perdido.

En cirugía bariátrica revisional, la alternativa más recomendada actualmente es el BGYR. La cirugía revisional, por ejemplo, extracción de BGA y conversión a BGYR, presenta una incidencia de complicaciones postoperatorias alta, del 10\% o más ${ }^{4}$.

Existe poca información y experiencia en relación al uso de la cirugía asistida por robot en procedimientos bariátricos revisionales.

En este estudio, de carácter transversal, presentamos nuestra experiencia inicial con cirugía bariátrica revisional asistida por robot, en pacientes de una institución de salud de Santiago de Chile.

\section{Material y Métodos}

Para este estudio, realizamos una revisión retrospectiva de todos los pacientes sometidos a pro- cedimientos bariátricos revisionales asistidos por robot, por un cirujano digestivo del Departamento de Cirugía Bariátrica y Departamento de Cirugía Robótica de la Clínica Santa María, en Santiago de Chile, entre agosto de 2013 y mayo de 2017. Todos los procedimientos fueron realizados con el mismo robot (DaVinci SI).

Se realizaron un total de 59 cirugías bariátricas revisionales asistidas por robot. Se registraron características demográficas de los pacientes, comorbilidades, indicación para la cirugía revisional, tiempo operatorio, pérdida estimada de sangre, tiempo de estadía hospitalaria, complicaciones postoperatorias y morbilidad a corto plazo ( $<30$ días).

\section{Resultados}

Un total de 59 pacientes fueron sometidos a cirugía bariátrica revisional asistida por robot.

La edad promedio fue de 47,8 años (29-65 años). Las características demográficas de los pacientes se encuentran descritas en la Tabla 1. El Índice de Masa Corporal (IMC) promedio al momento de la cirugía fue de $33,86 \mathrm{~kg} / \mathrm{m}^{2}$. Las indicaciones para cirugía revisional se encuentran resumidas

Tabla 1. Características demográficas de los pacientes

\begin{tabular}{|lc|}
\hline Número de pacientes & 59 \\
Género & \\
Masculino & $20(33,9 \%)$ \\
Femenino & $39(66,1 \%)$ \\
Edad promedio (años) & 47,8 \\
Comorbilidades & \\
Diabetes & $3(5,08 \%)$ \\
Prediabetes & $10(16,95 \%)$ \\
Sahos* & $3(5,08 \%)$ \\
Hipertensión arterial & $9(15,25 \%)$ \\
Colelitiasis/pólipos vesiculares & $7(11,86 \%)$ \\
Hígado graso & $10(16,95 \%)$ \\
Dislipidemia & $11(18,64 \%)$ \\
Hernia hiatal & $20(33,9 \%)$ \\
ERGE & $41(69,49 \%)$ \\
IMC promedio $\left(\mathrm{kg} / \mathrm{m}^{2}\right)$ & 33,86 \\
Peso promedio $(\mathrm{kg})$ & 94,3 \\
Tipo de cirugía bariátrica inicial & \\
BGA & $13(22,03 \%)$ \\
Gastrectomía en manga & $40(67,8 \%)$ \\
BGYR & $6(10,17 \%)$ \\
\hline
\end{tabular}

*Síndrome Apnea Obstructiva del Sueño. 
en la Tabla 2, siendo la indicación más frecuente la reganancia de peso y la enfermedad por reflujo gastroesofágico.

Se realizaron 59 cirugías bariátricas revisionales, 11 calibraciones o remodelamientos de gastrectomías en manga dilatadas, 34 BGYR con anastomosis manuales, 1 remodelamiento del pouch de BGYR, 3 extracciones de BGA, 1 con gastrectomía en manga simultánea, 5 instalaciones de BGA en pacientes con BGYR, 3 gastrectomías totales, 1 resección de fístula gastro-gástrica y 1 anastomosis gastroyeyunal (Tabla 3).

El tiempo operatorio promedio fue de $113,71 \mathrm{~min}$. El tiempo quirúrgico promedio fue de 101,63 min. La estadía hospitalaria promedio fue de 3,6 días. Fue necesario hospitalizar nuevamente a un paciente, que requirió otra cirugía secundaria a un biliperitoneo. Se observaron complicaciones postoperatorias en $3(5,08 \%)$ de los pacientes. Un paciente presentó una hemorragia digestiva alta autolimitada, que no requirió procedimientos adicionales. En los otros 2 $(3,39 \%)$ pacientes, se requirió una nueva intervención quirúrgica; un paciente cuya cirugía revisional consistió en conversión de una gastrectomía en manga en un BGYR, presentó un hemoperitoneo $24 \mathrm{~h}$ posterior a la cirugía. El otro paciente fue sometido a una conversión de gastrectomía en manga a BGYR, hernioplastía hiatal y colecistectomía, requirió una reintervención por un biliperitoneo, secundario a un conducto de Luchska no advertido durante la cirugía inicial. No se observaron fallecidos en esta serie de pacientes.

\section{Discusión}

Como se mencionó previamente, la cirugía bariátrica sigue siendo el único tratamiento efectivo a largo plazo para la baja de peso, sin embargo, han aumentado de forma importante las cirugías revisionales durante los últimos años, principalmente por la reganancia de peso y los efectos secundarios de estos procedimientos, especialmente, en pacientes con BGA y gastrectomía en manga.

A pesar de haber sido sometidos a procedimientos quirúrgicos bariátricos, en este grupo de pacientes, se observa un alto porcentaje de comorbilidades asociadas, especialmente hipertensión arterial, prediabetes y dislipidemia.

En los pacientes descritos en esta serie, la indicación más frecuente para realizar una cirugía revisional fue la reganancia de peso y la enfermedad por reflujo gastroesofágico (ERGE).

El procedimiento revisional más frecuente en esta serie fue el BGYR. En 3 pacientes se realizó una
Tabla 2. Indicaciones de cirugía revisional

\begin{tabular}{|lcc|}
\hline Indicación cirugía revisional & n de pacientes & $\begin{array}{c}\text { Porcentaje del total } \\
\text { de pacientes }\end{array}$ \\
\hline Reganancia de peso & 34 & $57,63 \%$ \\
\hline ERGE & 22 & $37,29 \%$ \\
\hline Fístula gástrica crónica & 2 & $3,39 \%$ \\
Desnutrición & 1 & $1,69 \%$ \\
\hline
\end{tabular}

Tabla 3. Cirugía revisional

\begin{tabular}{|lcc|}
\hline Cirugía revisional & n de pacientes & $\begin{array}{c}\text { Porcentaje del } \\
\text { total de pacientes }\end{array}$ \\
\hline Remodelamiento gastrectomía en manga & 11 & $18,65 \%$ \\
BGYR & 34 & $57,64 \%$ \\
\hline Remodelamiento BGYR & 1 & $1,69 \%$ \\
\hline Extracción BGA & 2 & $3,39 \%$ \\
\hline Extracción BGA y gastrectomía en manga & 1 & $1,69 \%$ \\
\hline BGA a BGYR & 5 & $8,48 \%$ \\
\hline Gastrectomía total & 3 & $5,08 \%$ \\
\hline Anastomosis gastroyeyunal & 1 & $1,69 \%$ \\
\hline Resección fístula gastro-gástrica & 1 & $1,69 \%$ \\
\hline
\end{tabular}

gastrectomía total como procedimiento revisional primario. En algunas ocasiones, las adherencias secundarias al procedimiento inicial, complicaciones postoperatorias como fístulas gástricas crónicas, generan un escenario de mucha dificultad, donde no existe otra alternativa que someter al paciente a procedimientos de mayor complejidad, como la gastrectomía total. El resultado en estos 3 pacientes fue positivo y efectivo. Ninguno presentó complicaciones postoperatorias.

El BGYR está asociado con un fracaso entre el 15 y $35 \%{ }^{5}$. La BGA laparoscópica en pacientes con BGYR previo es una técnica segura, que ha demostrado resultados efectivos en el porcentaje de pérdida de exceso de peso (PEP) en series pequeñas de pacientes, hasta un 38\% PEP con seguimiento a 1 año ${ }^{5}$.

Generalmente, los procedimientos revisionales están asociados a mayor morbilidad que las cirugías bariátricas iniciales. En esta serie de pacientes, la morbilidad fue baja, y no hubo mortalidad. Sólo 2 pacientes requirieron procedimientos adicionales, y un tercer paciente presentó una complicación autolimitada. A pesar de ser procedimientos que requieren de mayor tiempo, principalmente por adherencias 


\section{ARTÍCULO ORIGINAL}

abdominales, en esta serie, el tiempo quirúrgico promedio fue menor a $2 \mathrm{~h}$, incluyendo pacientes que fueron sometidos a cirugías de mayor complejidad y longitud, como gastrectomía total.

\section{Conclusión}

La cirugía bariátrica revisional asistida por robot es una técnica novedosa, que impresiona segura y efectiva. La cirugía robótica puede ser utilizada para procedimientos de alta complejidad, con baja morbilidad, baja mortalidad y buenos resultados. Se requieren series con mayor número de pacientes para confirmar lo que hemos observado en las experiencias iniciales, con respecto a los beneficios potenciales de esta técnica.

\section{Responsabilidades éticas}

Protección de personas y animales. Los autores declaran que para esta investigación no se han realizado experimentos en seres humanos ni en animales.

Confidencialidad de los datos. Los autores declaran que en este artículo no aparecen datos de pacientes.

Derecho a la privacidad y consentimiento informado. Los autores declaran que en este artículo no aparecen datos de pacientes.

\section{Conflicto de intereses}

Los autores declaran no tener ningún conflicto de intereses.

\section{Bibliografía}

1. Borbely Y, Winkler C, Kroll D, Nett P. Pouch Reshaping for Significant Weight Regain after Roux-en-Y Gastric Bypass. Obes Surg. 2017;27:439-44.

2. El Chaar M, Stoltzfus J, Claros L, Miletics M. Indications for Revisions Following 630 Consecutive
Laparoscopic Sleeve Gastrectomy Cases: Experience in a Single Accredited Center. J Gastrointest Surg. 2017;21:12-6.

3. Buchs N, Pugin F, Azagury D, Huber O, Chassot G, Morel P. Robotic revisional bariatric surgery: a comparative study with laparoscopic and open surgery. Int J Med Robotics Comput Assist Surg. 2015;11:284-9.
4. Wezenbeek M, Oudheusden T, Zoete J, Smulders J, Nienhuijs S. Conversion to Gastric Bypass After Either Failed Gastric Band or Failed Sleeve Gastrectomy. Obes Surg. 2017;27:83-9.

5. Uittenbogaart M, Leclercq W, Luijten A, MH van Dielen F. Laparoscopic Adjustable Gastric Banding After Failed Roux-En-Y Gastric Bypass. Obes Surg. 2017;27:381-6. 